Supporting Information (SI)

\title{
Facile Electrodeposition of Mn-CoP Nanosheets on Ni Foam as High-Rate and Ultrastable Electrodes for Supercapacitors
}

Miaomiao Zhang, ${ }^{\dagger}$ Haishun Du, ${ }^{\dagger}$ Zhen Wei, ${ }^{\ddagger}$ Xinyu Zhang ${ }^{*} \pitchfork$ Ruigang Wang ${ }^{*} \pitchfork$

$\dagger$ Department of Chemical Engineering, Auburn University, Auburn, Alabama 36849, United

States

$\$$ Department of Metallurgical and Materials Engineering, The University of Alabama, Tuscaloosa, Alabama 35487, United States

* E-mail addresses: xzz0004@auburn.edu.

*E-mail addresses: rwang@eng.ua.edu. 

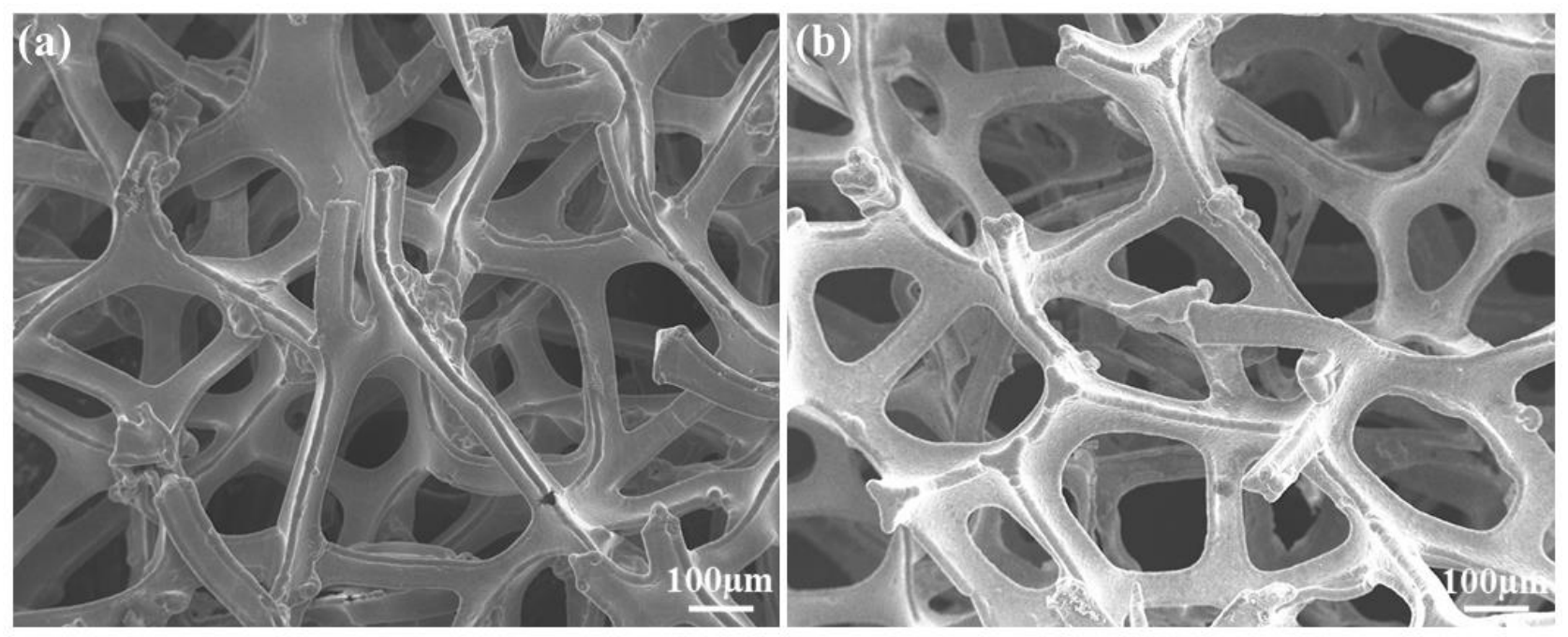

Figure S1. SEM images of (a) Ni foam and (b) Mn-CoP/NF.

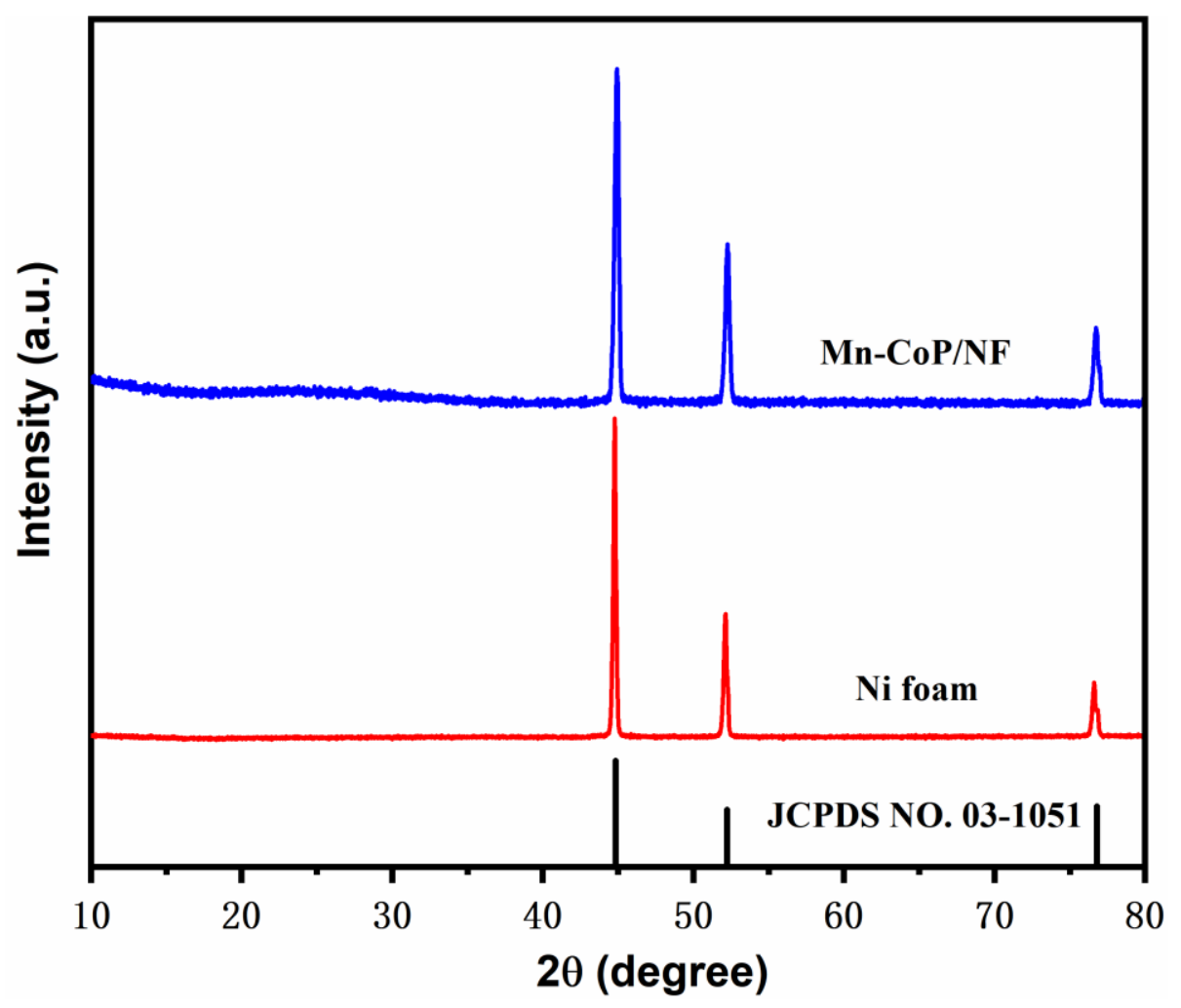

Figure S2. XRD patterns of Mn-CoP/NF and Ni foam. 